\title{
VCSEL-based sliceable bandwidth/bitrate variable transceivers
}

\author{
Michela Svaluto Moreolo*a, Laia Nadal ${ }^{\text {a }}$, Josep M. Fabrega ${ }^{\mathrm{a}}$, F. Javier Vilchez ${ }^{\text {a }}$, \\ Christian Neumeyr ${ }^{\mathrm{b}}$, Alberto Gatto ${ }^{\mathrm{c}}$, Paola Parolari ${ }^{\mathrm{c}}$, Pierpaolo Boffi ${ }^{\mathrm{c}}$ \\ ${ }^{a}$ Centre Tecnològic de Telecomunicacions de Catalunya (CTTC/CERCA), Av. C. F. Gauss 7, 08860 \\ Castelldefels (Barcelona), Spain; ' Vertilas GmbH, Daimlerstr. 11d, D-85748 Garching, Germany; \\ 'Politecnico di Milano, P.zza Leonardo da vinci 32, 20133, Milano, Italy
}

\begin{abstract}
The use of vertical cavity surface emitting laser (VCSEL) at long wavelengths, especially if characterized by large bandwidth or tunable capability, is appearing as an attractive technology for the implementation of advanced transceivers to be used in optical metro networks at $100 \mathrm{G}$ and beyond.

In this work, we report recent promising results on the adoption of different types of VCSEL for the sliceable bandwidth/bitrate variable transceiver (S-BVT) design. Special attention will be devoted to technological aspects and challenges, focusing on the added value of exploiting novel photonic technologies for the implementation of cost-effective transceivers, suitable for future optical metro networks targeting high capacity and flexibility.

Keywords: Sliceable bandwidth/bitrate variable transceiver (S-BVT), Vertical cavity surface emitting lasers (VCSEL), Future optical metro networks, Adaptive transmission, Multicarrier modulation (DMT/OFDM), Novel photonic technologies.
\end{abstract}

\section{INTRODUCTION}

Future optical metro networks pose several technological challenges to be urgently addressed for their high societal impact [1]. The high capacity demand, to support broadband services and everything-to-internet connectivity, in a dynamic scenario, while reducing the cost and power consumption, requires the aid of novel photonic technologies to be integrated within a flexible and programmable data plane [2].

In this context, the sliceable bandwidth/bitrate variable transceiver (S-BVT) represents a key element, to be suitably designed according to these constraints. Particularly, vertical cavity surface emitting laser (VCSEL) at long wavelengths is arising as a promising technology to be integrated in the transmitter design to reduce its cost, power consumption and footprint [3]. Recent results show that high capacity transmission (up to $100 \mathrm{~Gb} / \mathrm{s}$ with large bandwidth VCSELs) can be achieved, if combined with advanced modulation formats, including multicarrier modulation - such as discrete multitone (DMT) or orthogonal frequency division multiplexing (OFDM) - and 4-PAM [3-7]. Flexible rate and adaptive distance for metro network applications are enabled with bit/power loading algorithms and direct-modulated VCSEL-based S-BVT, attaining up to $200 \mathrm{~km}$ with direct detection (DD) [4]. By adopting coherent (CO) detection, the achievable reach can be further extended [7].

In addition, the adoption of widely (in a range of about $90 \mathrm{~nm}$ ) tunable micro-electro-mechanical system (MEMS) VCSEL also provides the S-BVT with advanced functionalities, enhancing the transceiver flexibility and tunability. This also includes the spectrum fragmentation mitigation $[3,4]$.

In this work, we report our results on the adoption of VCSEL technology for the S-BVT design, with special focus on the technological aspects, opportunities/benefits and limitations of this choice for future high capacity and agile optical metro networks.

The paper is organized as follows. Section 1 presents the long-wavelength VCSEL technology for both tunable MEMS VCSEL and large bandwidth VCSEL. Section 2 describes the architectures of S-BVT adopting tunable VCSEL with DD and large bandwidth VCSEL with CO-Rx, presenting the performance of both options. The results are discussed and finally Sec. 4 concludes the paper.

*michela.svaluto@cttc.es; phone +34 9364529 00; fax: +34 9364529 01; www.cttc.es 


\section{LONG-WAVELENGTH VCSEL TECHNOLOGY}

VCSEL consists of vertically stacked layers that allow the laser beam to be emitted vertically to the surface of the semiconductor device. The development of long-wavelength VCSELs is based on a laser design featuring a dielectric bottom mirror, a buried tunnel junction (BTJ) for the current confinement, a gold substrate acting as a heat sink, a multi quantum well (MQW) active region and an optimized waveguide design. The material structure consists of III-V semiconductors, such as In, P, Al, As and Ga, which enables the production of lasers operating at wavelengths ranging from $1.3 \mu \mathrm{m}$ up to $2.3 \mu \mathrm{m}$. Indium Phosphide (InP) long-wavelength VCSELs can be designed to allow laser operation from $-20^{\circ} \mathrm{C}$ to $+80^{\circ} \mathrm{C}$, providing excellent single mode performance with a side mode suppression ratio (SMSR) of $40 \mathrm{~dB}$ and more. Long-wavelength VCSELs features very efficient power consumptions with output optical powers up to $7 \mathrm{dBm}$ at bias currents in the range of 5-10 mA.

\subsection{Tunable VCSEL}

The single-mode VCSEL characterized by wide tunability in the C band is realized by a long-wavelength InP BTJ-based VCSEL and it features a MEMS top mirror [8], see Figure 1 (a). The small air gap between the surface of the base VCSEL and the MEMS can be thermo-electrically controlled, as can be seen in Figure 1 (b) where the operating wavelength is detailed as a function of both MEMS and VCSEL bias currents. The change in air gap leads to mode-hop free tuning of the laser wavelength of about $90 \mathrm{~nm}$ (from $1517 \mathrm{~nm}$ to $1608 \mathrm{~nm}$ ), showing a SMSR above $45 \mathrm{~dB}$ on the entire tuning range. The peak optical fiber-coupled power can be up to $1.2 \mathrm{~mW}$ at $22^{\circ} \mathrm{C}$ with a bias current of $32 \mathrm{~mA}$. The VCSEL has a maximum S-21 3-dB bandwidth of about $7 \mathrm{GHz}$ at $1550 \mathrm{~nm}$, while on a 47-nm wavelength range a minimum bandwidth of $4.5 \mathrm{GHz}$ is guaranteed [9].

\subsection{Large bandwidth short-cavity VCSEL}

Short-cavity (SC) VCSELs are designed to achieve large modulation bandwidths. To this purpose, it is beneficial to reduce the effective cavity length and the photon lifetime in the VCSEL cavity [10]. Therefore, the SC-VCSEL has two dielectric distributed Bragg reflectors (DBRs) featuring a short penetration depth of the optical field of only $410 \mathrm{~nm}$. Fig. 1 (c) depicts the SC-VCSEL structure: highly compressively strained Al-GaInAs/InGaAs quantum wells yield high (differential) gain and guarantee high relaxation-resonance frequencies, essential to achieve high modulation bandwidths. Small diameter $(<30 \mu \mathrm{m})$ of the semiconductor mesa, reduced contact-pad areas, and benzocyclobuthene spacer layers allow for the reduction of parasitic effects compromising high-speed modulation [11]. Typical SC devices present a current aperture of about $5 \mu \mathrm{m}$ and an effective cavity length of $2.5 \mu \mathrm{m}$ leading to modulation bandwidths up to $20 \mathrm{GHz}$. Finally, relative intensity noise below $-140 \mathrm{~dB} / \mathrm{Hz}$ can be obtained.

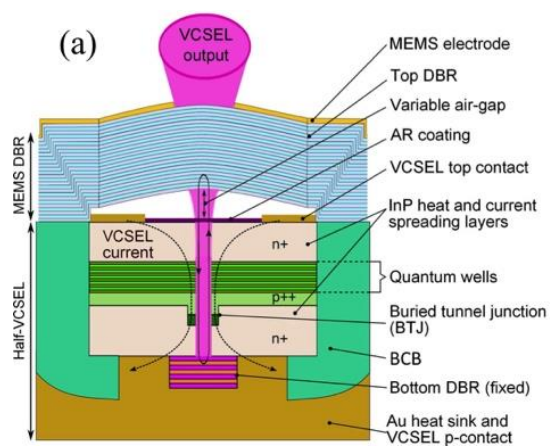

(b)

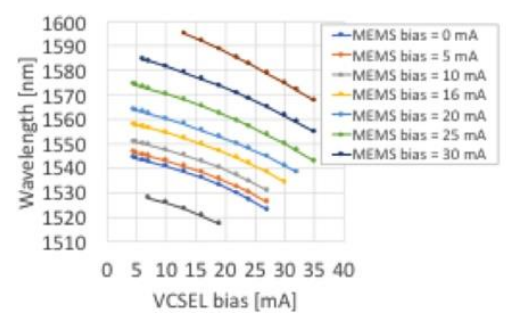

(c)

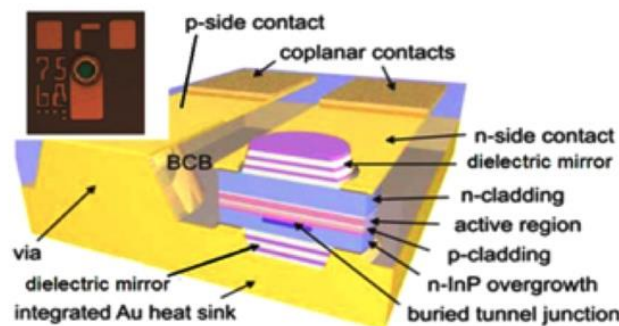

Figure 1. Structures of long-wavelength VCSEL: tunable featuring a MEMS top mirror (a) and large bandwidth SC (c). For the tunable VCSEL, operating wavelength as a function of MEMS and VCSEL bias currents (b).

\section{S-BVT BASED ON DIRECT MODULATED VCSEL}

Figure 2 describes a modular S-BVT architecture based on VCSEL technology, including alternative options for implementing the transmitter (Tx) and the receiver $(\mathrm{Rx})$ modules. Modularity allows enhancing the S-BVT capacity and features according to the need. The multiple modules at the Tx side of the S-BVT consists of direct modulated (DM) VCSELs (either tunable or large bandwidth SC). 
In case of tunable MEMS VCSEL, a single bitrate/bandwidth variable flow is generated at variable wavelength to be selected within the wide range of tunability available for this kind of technology (as presented in Sec 2). With a fine adjustment of MEMS, bias and temperature control (see Fig. 1(b)), the VCSEL can be tuned to operate within the C-band with flexgrid granularity $(6.25 \mathrm{GHz}$ and $12.5 \mathrm{GHz}$ frequency slots) and even finer [4]. Fig. 2 illustrates the corresponding BVT architecture. Multiple BVTs based on this approach can be integrated to implement an S-BVT. At the Rx side, two possible options are indicated: simple and cost-effective DD or CO detection for achieving enhanced performance. For this VCSEL type, we consider simple DD adopting a PIN receiver with a transimpedance amplifier (TIA).

When large bandwidth SC VCSEL are used for implementing the S-BVT, the wavelength cannot be arbitrarily selected within a wide range. Thus, in order to provide the module with a certain flexibility over the C-band spectrum for optical metro network applications, we consider sliceable bandwidth/bitrate variable transmitter (S-BVTx modules including a set of VCSEL with operating wavelengths opportunely spaced to cover the C-band. As considered in the framework of EU H2020 PASSION project [12], an integrated module including 40 VCSELs (4 sub-modules with 10 VCSELs each) covers the entire C-band with $100 \mathrm{GHz}$ granularity. By directly modulating each VCSEL (with $20 \mathrm{GHz}$ bandwidth) at $50 \mathrm{~Gb} / \mathrm{s}$, an aggregated capacity of up to $2 \mathrm{~Tb} / \mathrm{s}$ per Tx module is obtained, as shown in the (up-left) inset of Fig. 2. Multiple modules can generate an aggregated capacity of up to $8 \mathrm{~Tb} / \mathrm{s}$ (indicated in the inset as super module), enabling a total of $160 \mathrm{VCSELs}$ with channel spacing of $25 \mathrm{GHz}$. By suitably combining this transmitter architecture with CO-Rx modules, enhanced performance over the metro network can be achieved as shown in Sec. 3.2.

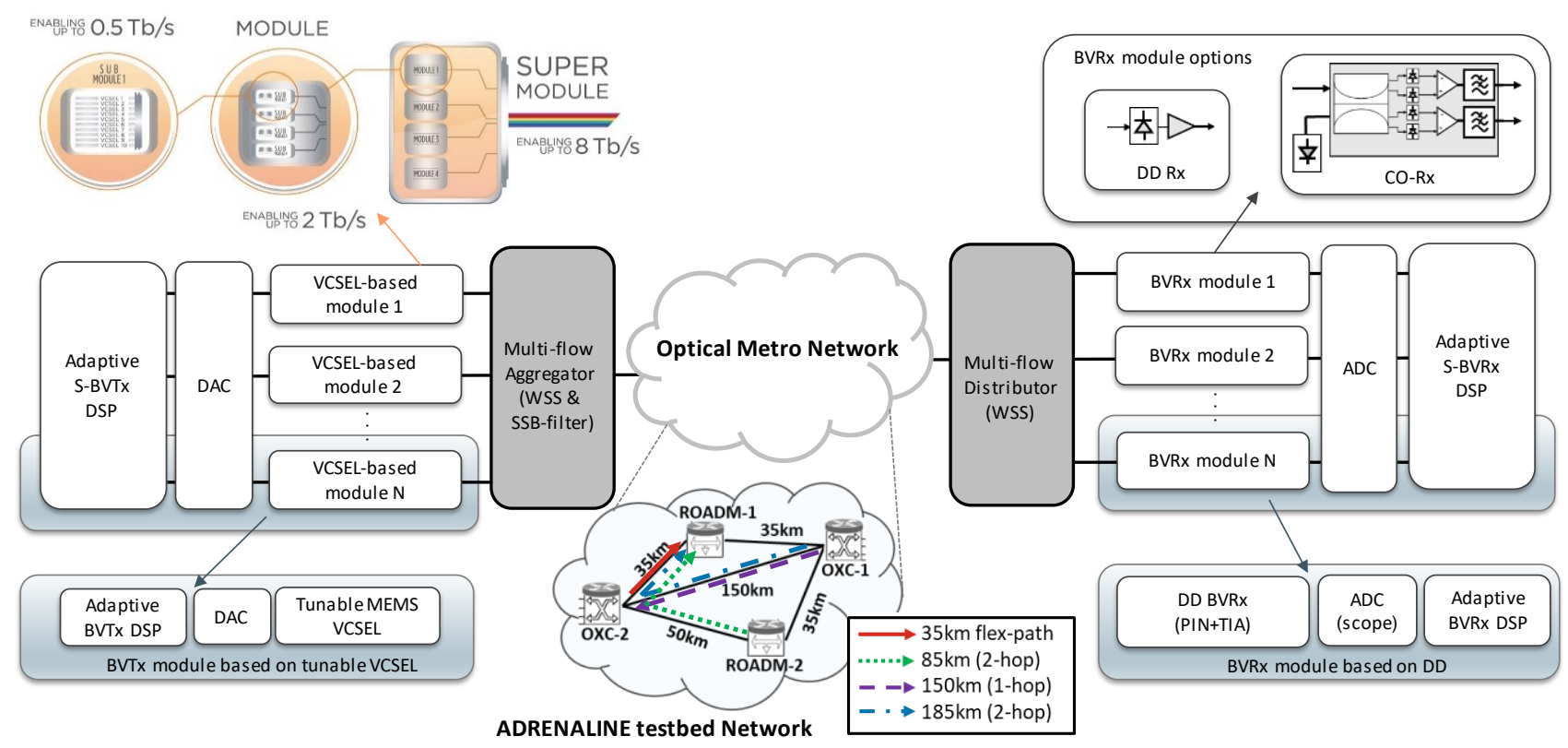

Figure 2. VCSEL-based S-BVT schematic; alternative module options in the insets at the Tx and Rx side. In the inset, the ADRENALINE testbed network is also depicted, including network paths analyzed for the experimental assessment of the BVT based on tunable MEMS VCSEL and DD.

\subsection{Performance using tunable VCSEL and DD}

Recently, tunable MEMS-VCSELs, with 3-dB bandwidth of $7 \mathrm{GHz}$, has been successfully demonstrated for different transmission systems and target applications. 10-Gb/s directly-modulated non-return-to-zero (NRZ) connection has been demonstrated for a compensated 40-km standard single mode fiber (SSMF) span [13]; while, 26 Gb/s DMT transmission over $40 \mathrm{~km}$ of SSMF has been reported for converged WDM-PON applications [5]. Similar performance has been also confirmed for DMT modulation with asymmetrical filtering [6].

This VCSEL technology has been also explored for implementing the building block, or specific modules, of an S-BVT able to cover extended reach, targeting the metro segment $[3,4]$. In particular, single sideband (SSB) OFDM modulation has been considered to enhance the robustness against chromatic dispersion. SSB optical filtering can be performed at the S-BVT aggregator, or at the network nodes, if equipped with bandwidth variable wavelength selective switch (WSS) elements. 
To assess the performance in terms of impairments tolerance and capacity, of a BVT module adopting a widely tunable MEMS VCSEL, SSB-OFDM has been compared to pure DMT transmission [2]. The VCSEL-based BVTx is combined with a pre-amplified DD receiver (PIN with TIA) at the BVRx. The adaptive DSP enables rate/distance and bandwidth variable transmission thanks to the multicarrier modulation (512 subcarriers over $16 \mathrm{GHz})$ and Levin-Campello loading algorithm [14]. The maximum bandwidth with assigned bits is found to be $10.5 \mathrm{GHz}$, considering the VCSEL bandwidth and the signal-to-noise ratio (SNR) values retrieved at the BVRx, probing the channel state information (see Fig. 3 (a) and (b)). Thus, in case of DMT, the optical spectrum occupancy is $25 \mathrm{GHz}$ (in terms of assigned flexgrid frequency slots of $12.5 \mathrm{GHz}$ ), while for the SSB-OFDM only one frequency slot of $12.5 \mathrm{GHz}$ is required. The digital signals are converted to analog with a 64GSa/s digital-to-analog converter (DAC). At the BVRx, the photodetected signal is captured by an oscilloscope at 100GSa/s for demodulation and error counting. As shown in Fig. 3 (c), the maximum back-to-back (B2B) capacity, below the target BER threshold set to $4.62 \cdot 10^{-3}$ (7\% overhead over a total of $13.4 \%$ ), is $33.2 \mathrm{~Gb} / \mathrm{s}$ for DMT and $31.7 \mathrm{~Gb} / \mathrm{s}$ for SSB-OFDM, at $1550.12 \mathrm{~nm}$. Similar performance has been found also at different operating wavelengths (at the edge of the ITU grid: $1530.33 \mathrm{~nm}$ and $1561.42 \mathrm{~nm}$ ) [4]. Fig. 3 (d) reports the capacity as a function of the optical SNR (OSNR) values measured in the B2B case for DMT.

(a)

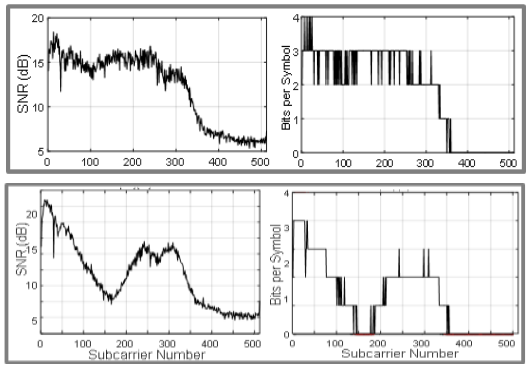

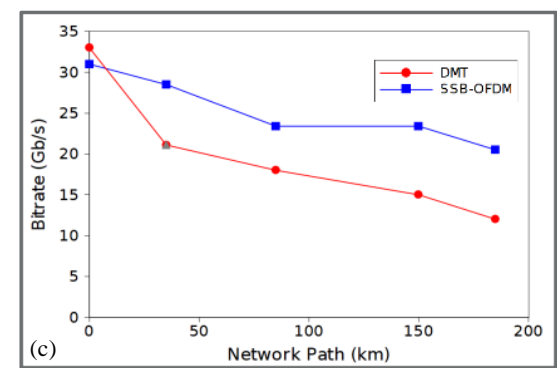

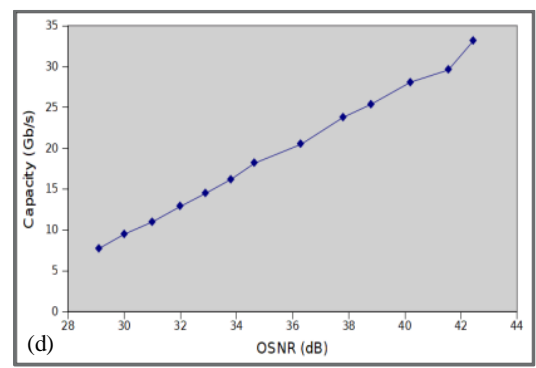

Figure 3. SNR values retrieved at the BVRx after $35 \mathrm{~km}$ path and corresponding bit assignment per (a) SSB-OFDM and (b) DMT subcarrier; BVT capacity performance versus (c) ADRENALINE testbed network paths and (d) OSNR measured in B2B for DMT.

The assessment has been also performed over a real testbed: the ADRENALINE network testbed consisting of four nodes (two optical cross-connects, OXCs, and two reconfigurable optical add-drop multiplexers, ROADMs) and five amplified links ranging from $35 \mathrm{~km}$ to $150 \mathrm{~km}$ (see inset of Fig. 2) [15]. All the links are SSMF but the $150 \mathrm{~km}$ link, which is a nonzero dispersion-shifted fiber (NZDSF). Different paths have been considered for the experimental demonstration: a flexgrid path of $35 \mathrm{~km}$ (between ADRENALINE OXC-2 and ROADM-1 equipped with programmable WSS), a single-hop path of $150 \mathrm{~km}$, and 2-hop paths of $85 \mathrm{~km}(50 \mathrm{~km}+35 \mathrm{~km})$ and of $185 \mathrm{~km}(150 \mathrm{~km}+35 \mathrm{~km})$, respectively. Performance reported in Fig. 3 (c), and summarized in Table 1, shows that SSB-OFDM outperforms DMT for all the analyzed paths, supporting connections above $20 \mathrm{~Gb} / \mathrm{s}$ at the target BER over the longest one (2-hop of 185km). Being more severely affected by chromatic dispersion, DMT is characterized by poorer transmission performance. It is interesting to notice that in case of SSB-OFDM, similar performance is achieved over the 150km NZDSF single-hop path and traversing the 2 hops of the 85 km SSMF path.

Table 1 Capacities of tunable VCSEL (at $1550.12 \mathrm{~nm}$ ) BVT considering DMT (25GHz spectral occupancy) or SSB-OFDM (halfbandwidth) and DD for different (ADRENALINE testbed) network paths (B2B case as reference)

\begin{tabular}{|c|c|c|c|c|c|}
\hline & B2B & $\begin{array}{c}\text { Reach }=\mathbf{3 5 k m} \\
\text { (flexgrid } \\
\text { single-hop) }\end{array}$ & $\begin{array}{c}\text { Reach }=\mathbf{8 5 k m} \\
\text { (2-hop: } \\
50 \mathrm{~km}+35 \mathrm{~km})\end{array}$ & $\begin{array}{c}\text { Reach = 150km } \\
\text { (single-hop) }\end{array}$ & $\begin{array}{c}\text { Reach = 185km } \\
\text { (2-hop: } \\
150 \mathrm{~km}+35 \mathrm{~km})\end{array}$ \\
\hline DSB-DMT & $33.2 \mathrm{~Gb} / \mathrm{s}$ & $21.1 \mathrm{~Gb} / \mathrm{s}$ & $18 \mathrm{~Gb} / \mathrm{s}$ & $15 \mathrm{~Gb} / \mathrm{s}$ & $12 \mathrm{~Gb} / \mathrm{s}$ \\
\hline SSB-OFDM & $31.7 \mathrm{~Gb} / \mathrm{s}$ & $28.5 \mathrm{~Gb} / \mathrm{s}$ & $23.4 \mathrm{~Gb} / \mathrm{s}$ & $23.4 \mathrm{~Gb} / \mathrm{s}$ & $20.5 \mathrm{~Gb} / \mathrm{s}$ \\
\hline
\end{tabular}




\subsection{Performance using large bandwidth SC VCSEL and CO-Rx}

In order to evaluate the performance of metro networks employing VCSEL-based transmitters (S-BVT), enabling high capacity and extended reach, we have performed some simulations exploiting the large bandwidth of SC VCSEL and CO detection.

The adoption of DM of the optical source with a multicarrier signal, such as DMT, allows the use of a simplified coherent receiver [7]. In fact, the transmitted signal is just intensity modulated: after I and Q components recovery and chromatic dispersion (CD) compensation the I and Q square moduli are provided and summed up in order to obtain the originally transmitted intensity signal. This approach avoids the use of phase and frequency recovery, reducing the complexity of the receiver DSP and also relaxing the constraints on VCSEL and local oscillator (LO) linewidths. As a drawback this operation cancels the advantages in terms of bit error rate (BER) as a function of SNR of CO detection.

In our simulations, the $\mathrm{CO}$ receiver exploits a $10 \mathrm{dBm}$ per polarization LO with $100-\mathrm{kHz}$ linewidth and has $25 \mathrm{GHz}$ electrical bandwidth. Single polarization, single channel performance with DMT modulation is evaluated thus the receiver DSP provides CD compensation, digital symbol synchronization, cyclic prefix (CP) removal, subcarriers phase recovery, demodulation and error count. In the following the transmitted capacity is evaluated performing Chow's bit loading algorithm [16] with a target BER of $3.8 \times 10^{-3}$. As the transmitter is concerned, we model the employed VCSELs with 5 $\mathrm{MHz}$ linewidth, a chirp factor $\alpha=3$ and a modulation frequency bandwidth of $18 \mathrm{GHz}$. The adopted DMT signal is obtained with expected bias current and modulation depth, of $8 \mathrm{~mA}$ and $12 \mathrm{~mA}$ respectively. In particular, we evaluated two DMT signals occupying an optical spectrum of less than $25 \mathrm{GHz}$, in order to fit a $25 \mathrm{GHz}$ minimum WDM channel spacing. We considered DSB and SSB transmissions; the DSB DMT signal is composed by 256 subcarriers in $10 \mathrm{GHz}$ range, i.e. the subcarrier spacing is $39.062 \mathrm{MHz}$, occupying an optical bandwidth of around $20 \mathrm{GHz}$; a cyclic prefix (CP) of about $2.1 \%$ of the symbol length is also added. On the other hand, the SSB transmission is performed by properly detuning a WSS filter of $21 \mathrm{GHz}$ full width half maximum (FWHM) [17] with respect to the carrier of a DMT signal composed by 256 subcarriers in $20 \mathrm{GHz}$ range, i.e. the subcarrier spacing is $78.125 \mathrm{MHz}$; again a $\mathrm{CP}$ of about $2.1 \%$ of the symbol length is added. Transmission performance is analyzed through SSMF propagation, with $0.25 \mathrm{~dB} / \mathrm{km}$ attenuation and $17 \mathrm{ps} / \mathrm{nm} \mathrm{km}$ dispersion at $1550 \mathrm{~nm}$; fibre spans of $35 \mathrm{~km}$ and $65 \mathrm{~km}$ are taken into account with the employment of Erbium-doped fibre amplifiers (EDFAs) with $6 \mathrm{~dB}$ noise figure (NF) to compensate for the transmission losses of each span. Moreover, the effect of cascading filtering due to node presence is taken into account by the number of traversed WSS filters, previously described. Figure 4 shows the obtained transmission capacity as a function of the number of traversed filters for OSNR values between $25 \mathrm{~dB}$ and $40 \mathrm{~dB}$, in case of SSB or DSB DMT.
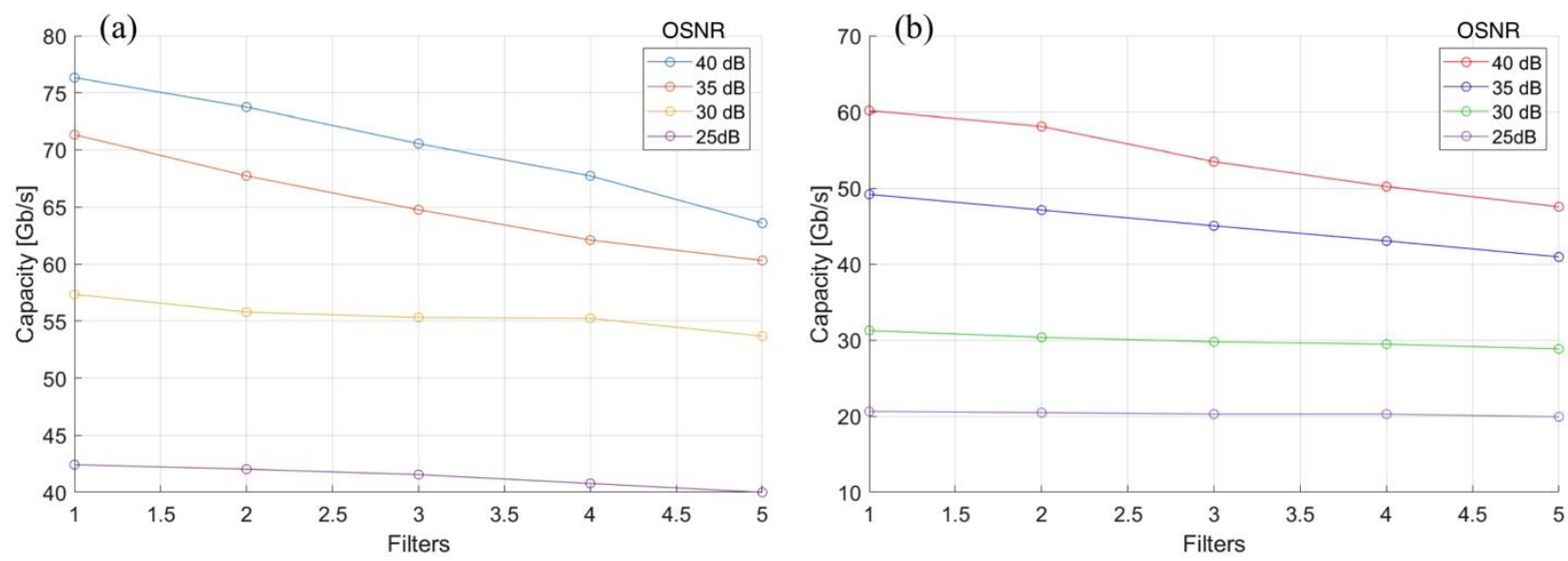

Figure 4. Capacity as a function of the number of WSS for OSNR ranging from $25 \mathrm{~dB}$ to $40 \mathrm{~dB}$ : (a) SSB DMT modulation with electrical signal occupancy of $20 \mathrm{GHz}$, (b) DSB DMT modulation with electrical signal occupancy of $10 \mathrm{GHz}$.

Tables 2 and 3 display the performance in terms of transmitted capacity and maximum achievable reach in case of either $35 \mathrm{~km}$ or $65 \mathrm{~km}$ SSMF span length as a function of OSNR. Table 2 presents DMT with DSB modulation performance as a reference, and SSB-DMT modulation after crossing up to 5 filters (WSS) and considering different OSNR values. In both cases the signal bandwidth occupancy is $20 \mathrm{GHz}$. SSB is obtained by exploiting a WSS detuning of $9.5 \mathrm{GHz}$ with respect to the VCSEL carrier. The considered transmitted power is $0 \mathrm{dBm}$. 
Table 2 Capacities for single-channel, single-polarization transmission for DSB and SSB DMT modulation with electrical signal occupancy of $20 \mathrm{GHz}$

\begin{tabular}{|c|c|c|c|c|c|}
\hline & $\begin{array}{c}\text { OSNR }=\mathbf{4 0} \mathbf{~ d B} \\
\text { Reach }_{35 \mathrm{~km}}=\mathbf{7 0 k m} \\
\text { Reach }_{65 \mathrm{~km}}=\mathbf{n . a} .\end{array}$ & $\begin{array}{c}\text { OSNR }=\mathbf{3 5} \mathbf{~ d B} \\
\text { Reach }_{35 \mathrm{~km}}=\mathbf{2 1 0 k m} \\
\text { Reach }_{65 \mathrm{~km}}=\mathbf{6 5} \mathbf{~ k m}\end{array}$ & $\begin{array}{c}\text { OSNR }=\mathbf{3 0 ~ d B} \\
\text { Reach }_{35 \mathrm{~km}}=\mathbf{7 3 5} \mathbf{k m} \\
\text { Reach }_{65 \mathrm{~km}}=\mathbf{2 6 0 \mathrm { km }}\end{array}$ & $\begin{array}{c}\text { OSNR }=\mathbf{2 9} \mathbf{~ d B} \\
\text { Reach }_{35 \mathrm{~km}}=\mathbf{9 4 5} \mathbf{k m} \\
\text { Reach }_{65 \mathrm{~km}}=\mathbf{3 2 5 k m}\end{array}$ & $\begin{aligned} \text { OSNR } & =\mathbf{2 5} \mathbf{~ d B} \\
\text { Reach }_{35 \mathrm{~km}} & =\mathbf{2 3 1 0} \mathbf{k m} \\
\text { Reach }_{65 \mathrm{~km}} & =\mathbf{7 8 0} \mathrm{km}\end{aligned}$ \\
\hline $\begin{array}{c}\text { DSB w/o WSS } \\
\left(B_{\text {signal }}=20 \mathrm{GHz}\right)\end{array}$ & $96 \mathrm{~Gb} / \mathrm{s}$ & $68 \mathrm{~Gb} / \mathrm{s}$ & $44 \mathrm{~Gb} / \mathrm{s}$ & $41 \mathrm{~Gb} / \mathrm{s}$ & $18 \mathrm{~Gb} / \mathrm{s}$ \\
\hline SSB w 1 WSS & $76 \mathrm{~Gb} / \mathrm{s}$ & $71 \mathrm{~Gb} / \mathrm{s}$ & $57 \mathrm{~Gb} / \mathrm{s}$ & $54 \mathrm{~Gb} / \mathrm{s}$ & $42 \mathrm{~Gb} / \mathrm{s}$ \\
\hline SSB w 2 WSS & $74 \mathrm{~Gb} / \mathrm{s}$ & $68 \mathrm{~Gb} / \mathrm{s}$ & $56 \mathrm{~Gb} / \mathrm{s}$ & $53 \mathrm{~Gb} / \mathrm{s}$ & $42 \mathrm{~Gb} / \mathrm{s}$ \\
\hline SSB w 5 WSS & $64 \mathrm{~Gb} / \mathrm{s}$ & $60 \mathrm{~Gb} / \mathrm{s}$ & $54 \mathrm{~Gb} / \mathrm{s}$ & $51 \mathrm{~Gb} / \mathrm{s}$ & $40 \mathrm{~Gb} / \mathrm{s}$ \\
\hline
\end{tabular}

Table 3 shows the obtained results in terms of transmitted capacity and maximum reach for different OSNR values and after crossing up to 5 filters (WSS). A DSB DMT signal with $10 \mathrm{GHz}$ bandwidth occupancy (optical spectrum width around $20 \mathrm{GHz}$ ) and either $35 \mathrm{~km}$ or $65 \mathrm{~km}$ SSMF spans are considered. Again, the transmitted power is $0 \mathrm{dBm}$.

Table 3 Capacities for single-channel, single-polarization transmission for DSB DMT modulation with electrical signal occupancy of $10 \mathrm{GHz}$

\begin{tabular}{|c|c|c|c|c|}
\hline & $\begin{array}{c}\text { OSNR }=40 \mathrm{~dB} \\
\text { Reach } 35 \mathrm{~km}=\mathbf{7 0} \mathbf{~ k m} \\
\text { Reach }_{65 \mathrm{~km}}=\mathbf{n . a}\end{array}$ & $\begin{array}{c}\text { OSNR }=35 \mathrm{~dB} \\
\text { Reach } 35 \mathrm{~km}=\mathbf{2 1 0} \mathbf{~ k m} \\
\text { Reach } 65 \mathrm{~km}=\mathbf{6 5} \mathbf{~ k m}\end{array}$ & $\begin{array}{c}\text { OSNR }=30 \mathrm{~dB} \\
\text { Reach } 35 \mathrm{~km}=\mathbf{7 3 5} \mathbf{~ k m} \\
\text { Reach } 65 \mathrm{~km}=\mathbf{2 6 0} \mathbf{~ k m}\end{array}$ & $\begin{array}{c}\text { OSNR }=25 \mathrm{~dB} \\
\text { Reach } 35 \mathrm{~km}=\mathbf{2 3 1 0} \mathbf{~ k m} \\
\text { Reach } 65 \mathrm{~km}=\mathbf{7 8 0} \mathbf{~ k m}\end{array}$ \\
\hline $\begin{array}{c}\text { DSB w/o WSS } \\
\left(\text { B }_{\text {signal }}=10 \mathrm{GHz}\right)\end{array}$ & $64 \mathrm{~Gb} / \mathrm{s}$ & $50 \mathrm{~Gb} / \mathrm{s}$ & $31 \mathrm{~Gb} / \mathrm{s}$ & $20 \mathrm{~Gb} / \mathrm{s}$ \\
\hline DSB w 1 WSS & $59 \mathrm{~Gb} / \mathrm{s}$ & $48 \mathrm{~Gb} / \mathrm{s}$ & $30 \mathrm{~Gb} / \mathrm{s}$ & $20 \mathrm{~Gb} / \mathrm{s}$ \\
\hline DSB w 2 WSS & $55 \mathrm{~Gb} / \mathrm{s}$ & $46 \mathrm{~Gb} / \mathrm{s}$ & $30 \mathrm{~Gb} / \mathrm{s}$ & $20 \mathrm{~Gb} / \mathrm{s}$ \\
\hline DSB w 5 WSS & $47 \mathrm{~Gb} / \mathrm{s}$ & $41 \mathrm{~Gb} / \mathrm{s}$ & $28 \mathrm{~Gb} / \mathrm{s}$ & $20 \mathrm{~Gb} / \mathrm{s}$ \\
\hline
\end{tabular}

\subsection{Discussion of the results}

Adopting DM tunable VCSELs and DD, the BVT capacity in B2B with optimized BL/PL assignment and $10.5 \mathrm{GHz}$ bandwidth occupancy is above $30 \mathrm{~Gb} / \mathrm{s}$. However, this capacity decreases with the increase of the link distance due to the chromatic dispersion effect. Thanks to the use of SSB-OFDM, which is more robust against chromatic dispersion, the reach can be extended to support $20 \mathrm{~Gb} / \mathrm{s}$ connections over metro network path of $185 \mathrm{~km}$ with 2 hops. In case of using DMT, which requires the double of spectral resource to transmit the same data flow, only $12 \mathrm{~Gb} / \mathrm{s}$ can be supported. The results also show that, as expected, traversing multiple hops has an impact on the capacity performance. This aspect has been more extensively analyzed in case of using (S)-BVT adopting large bandwidth SC VCSEL and CO-Rx: the effect of cascading multiple WSS (up to 5 have been considered) degrades the performance and this effect is more pronounced for short distances. In fact, it is interesting to note that for the longest analyzed reach this effect is negligible for SSB and in case of DSB is not evidenced at all. Also, it can be observed that the reach is extended (up to a factor of 3), considering shorter SSMF span $(35 \mathrm{~km}$ instead of $65 \mathrm{~km}$ ). Furthermore, it has been shown that $50 \mathrm{~Gb} / \mathrm{s}$ connections with frequency channels of $25 \mathrm{GHz}$ can be supported over a path above $900 \mathrm{~km}$ (traversing 5 WSSes), considering SSB SSMF span of 35 $\mathrm{km}$ and $29 \mathrm{~dB}$ OSNR. The reach can be further extended by a factor of 2.4, lowering the bit rate to $40 \mathrm{~Gb} / \mathrm{s}$ for $25 \mathrm{~dB}$ OSNR. Considering only one WSS, for example the one at the S-BVT aggregator, up to $70 \mathrm{~Gb} / \mathrm{s}$ connections can be supported over a path of $200 \mathrm{~km}$.

\section{CONCLUSIONS}

The presented results are definitively promising for introducing VCSEL as key enabling technology for the implementation of sliceable bandwidth/bitrate variable transceivers in future high capacity and agile optical metro networks. Particularly, tunable VCSEL can be combined with DD to offer adaptive capacities in the range 30-10 Gb/s over network paths up to 
$200 \mathrm{~km}$, with high flexibility in the operating wavelength selection. In order to achieve ultimate performance in terms of capacity and reach, larger bandwidth VCSELs can be adopted and conveniently combined with coherent detection. This enables to extend the achievable reach at the expenses of limiting the tunability range. The presented results show that up to $50 \mathrm{~Gb} / \mathrm{s}$ connections per flow can be enabled over hundreds of $\mathrm{km}$ adopting SSB modulation and adaptive DSP. S-BVT aggregating multiple flows can further enhance the capacity to support multi-terabit connections in order to address the needs of future metro networks.

\section{ACKNOWLEDGMENT}

This paper is part of a project that has received funding from the European Union's Horizon 2020 research and innovation programme under grant agreement PASSION No 780326. This work has been also partially supported by the Spanish DESTELLO (TEC2015-69256-R) and AURORAS (RTI2018-099178-B-I00) projects.

\section{REFERENCES}

[1] Svaluto Moreolo M., Fabrega J. M., Nadal L., " S-BVT for next-generation optical metro networks: benefits, design and key enabling technologies," Proc. SPIE 10129, San Francisco, CA (USA), (2017).

[2] Svaluto Moreolo M., Fabrega J. M., Nadal L., "Programmable Optical Transmission Systems in the Hyperconnectivity Era: A Synergy of Photonic Technologies and Software-Defined Networking," Proc. OSA Advanced Photonics Congress (APC, NETWORKS), Zurich (Switzerland), (2018).

[3] Svaluto Moreolo M., Fabrega J. M., Nadal L., Vilchez F. J., " Exploring the Potential of VCSEL Technology for Agile and High Capacity Optical Metro Networks," Proc. ONDM, Dublin, Ireland, (2018).

[4] Svaluto Moreolo M., et al., "Modular SDN-enabled S-BVT Adopting Widely Tunable MEMS VCSEL for Flexible/Elastic Optical Metro Networks," Proc. OFC, S. Diego, CA (USA), (2018).

[5] Wagner C., et al., "26-Gb/s DMT Transmission Using Full C-Band Tunable VCSEL for Converged PONs," Photonic technology Lett., vol. 29, no. 17, pp. 1475 - 1478, (2017).

[6] Gatto A., Parolari P., Neumeyr C. and Boffi P., "Beyond 25 Gb/s Directly-Modulated Widely Tunable VCSEL for Next Generation Access Network," Proc. OFC, San Diego, CA, (2018).

[7] Xie, C. et al., "400-Gb/s PDM-4PAM WDM System Using a Monolithic $2 \times 4$ VCSEL Array and Coherent Detection," J. Lightwave Technol. 33, 670-677 (2015).

[8] Paul S., et al., "High speed surface micromachined MEMS tunable VCSEL for telecom wavelengths," proc. CLEO, AM3K.1, San Jose (2015).

[9] Paul S., et al., "10-Gb/s Direct Modulation of Widely Tunable 1550-nm MEMS VCSEL”, IEEE J. Sel. Top. Quant. Electr., Vol. 21, No. 6, p. 1700908 (2015)

[10] Westbergh P., Gustavsson J. S., Kogel B., Haglund A., Larsson A., "Impact of photon lifetime on high-speed VCSEL performance,” IEEE J. Sel. Topics Quantum Electron., vol. 17, no. 6, pp. 1603-1613, (2011)

[11] Mueller M., et al., “1550 nm highspeed short-cavity VCSELs,” IEEE J. Sel. Topics Quantum Electron., vol. 17, no. 5, pp. 1158-1166, (2011)

[12] http://www.passion-project.eu/

[13] Wagner C., et al., "Full C-band Tunable MEMS-VCSEL for Next Generation G.metro Mobile Front- and Backhauling," Proc. OFC, W2A.27, Los Angeles (2017).

[14] Nadal L., et al., "DMT Modulation with Adaptive Loading for High Bit Rate Transmission Over Directly Detected Optical Channels," J. Lightwave Technol., vol. 32, no. 21, pp. 3541-3551 (2014).

[15] Muñoz R., et al., "The ADRENALINE Testbed: An SDN/NFV Packet/Optical Transport Network and Edge/Core Cloud Platform for End-to-End 5G and IoT Services," Proc. EuCNC, (2017).

[16] Chow, P. S. et al., "A practical discrete multitone transceiver loading algorithm for data transmission over spectrally shaped channels," IEEE Trans. on comm., 43, 773-775, (1995).

[17] Pulikkaseril, C., et al., "Spectral modeling of channel band shapes in wavelength selective switches," Opt. Express $19,8458-8470$ (2011). 